\title{
MULTIPLIERS OF SEGAL ALGEBRAS
}

\author{
U. B. TEWARI
}

\begin{abstract}
We show that there exists a noncompact locally compact abelian group $G$ and a Segal algebra $S(G)$ on $G$ whose multiplier algebra properly contains the measure algebra.
\end{abstract}

1. Introduction. Let $G$ be a locally compact abelian group and let $S_{1}(G)$, $S_{2}(G)$ be two Segal algebras on $G$ (for definitions, see Reiter [8]). A multiplier of $S_{1}(G)$ to $S_{2}(G)$ is a linear operator $T$ on $S_{1}(G)$ to $S_{2}(G)$ such that $T(f * g)=f * T g$ for each $f \in L^{1}(G)$ and $g \in S_{1}(G)$. It is well known that if $T$ is a multiplier of $S_{1}(G)$ to $S_{2}(G)$, then there exists a unique bounded continuous function $\phi$ on $\hat{G}$, the dual group of $G$, such that $\widehat{T f}(\gamma)=\phi(\gamma) \hat{f}(\gamma)$ for every $f \in S_{1}(G)$ and $\gamma \in \hat{G}$. Conversely, if $\phi$ is a bounded continuous function on $\hat{G}$ such that for every $f \in S_{1}(G)$ there exists a $g \in S_{2}(G)$ such that $\hat{g}(\gamma)=\phi(\gamma) \hat{f}(\gamma)$ for every $\gamma \in \hat{G}$, then there exists a multiplier $T$ of $S_{1}(G)$ to $S_{2}(G)$ such that $\widehat{T f}(\gamma)=\phi(\gamma) \hat{f}(\gamma)$ for every $f \in S_{1}(G)$ and $\gamma \in \hat{G}$. The function $\phi$, associated with the multiplier $T$, is called the Fourier transform of $T$ and is denoted by $\hat{T}$, so that $\hat{T}(\gamma)=\phi(\gamma)$ for every $\gamma \in \hat{G}$. The space of multipliers of $S_{1}(G)$ to $S_{2}(G)$ is denoted by $M\left(S_{1}(G), S_{2}(G)\right)$. If $S(G)$ is a Segal algebra, a multiplier of $S(G)$ to $S(G)$ is called a multiplier of $S(G)$ and the space $M(S(G), S(G))$ is denoted by $M(S(G))$. The space $M(S(G))$ is a Banach algebra with the norm as operator norm and the multiplication as composition of operators. The Banach algebra $M(S(G))$ is called the multiplier algebra of $S(G)$. For notations not explained here and the standard results from harmonic analysis, the reader is referred to Rudin [9, Chapter 1]. For a study of $M(S(G))$ from a general point of view, see [1].

If $G$ is noncompact, then for every Segal algebra $S(G)$ on $G$ whose multipliers have been described in the literature, it has been found that $M(S(G))$ is isomorphic with $M(G)$, the measure algebra on $G$. This leads one to conjecture that for noncompact $G$ and any Segal algebra $S(G)$ on $G$, $M(S(G))$ is always isomorphic to $M(G)$. In this regard the following remark due to H. C. Lai, in an Autoreferat to one of his papers (Zentralblatt 235, 43007), is interesting:

"It can be remarked here that the multipliers of a Segal algebra $S(G)$ for noncompact $G$ is also isometrically isomorphic to $M(G)$ by the same arguments as the proof for $A^{p}(G)$."

In this paper, we shall see that the above-mentioned conjecture is false and, consequently, the remark of H. C. Lai is not justified. After we wrote this

Received by the editors February 20, 1975.

AMS (MOS) subject classifications (1970). Primary 43A22; Secondary 42A18.

Key words and phrases. Locally compact abelian group, Segal algebra, multiplier algebra, measure algebra, tensor product. 
paper, we were informed by R. R. Goldberg that H. Krogstad has shown that the multiplier algebra of the Wiener algebra $M_{1}(R)$, on the real line, properly contains the measure algebra. Thus the above conjecture is proved to be false. The proof in this paper does not involve the Wiener algebra and depends on some abstract arguments which appear to be interesting. We also prove a result about the multipliers of the Wiener algebra showing that the multiplier algebra of $M_{1}(R)$ is a proper subset of the set of multipliers of $M_{1}(R)$ to $L^{1}(R)$.

2. Preliminaries. For obtaining our results and a proper understanding of the proofs we shall need the following results about the projective tensor products of Segal algebras. We shall not attempt to define the projective tensor products as it is well known. The results about group algebras can be seen in Johnson [4] and the results about Segal algebras are contained in Kapoor [5].

Let $G$ and $H$ be locally compact abelian groups. For $a \in L^{1}(G)$ and $b \in L^{1}(H)$, we define $a \cdot b(x, y)=a(x) \cdot b(y)$ for $(x, y) \in G \times H$. The following two results can be found in [4].

I. There exists an isometry $T: L^{1}(G) \otimes_{\gamma} L^{1}(H) \rightarrow L^{1}(G \times H)$ which carries $a \otimes b \rightarrow a \cdot b$.

II. The maximal ideal space of $L^{1}(G) \otimes_{\gamma} L^{1}(H)$ can be identified with $\hat{G} \times \hat{H}$ in such a way that $T \hat{f}(\hat{g}, \hat{h})=\hat{f}(\hat{g}, \hat{h})$ for $f \in L^{1}(G) \otimes_{\gamma} L^{1}(H)$ and $(\hat{g}, \hat{h}) \in \hat{G} \times \hat{H}$.

Let $S(G)$ and $S(H)$ be Segal algebras on $G$ and $H$ respectively. For $s \in S(G)$ and $t \in S(H), s \otimes t$ will be considered as an element of $S(G) \otimes_{\gamma}$ $S(H)$ as well as an element of $L^{1}(G) \otimes_{\gamma} L^{1}(H)$. The next result is from [5].

III. The map $I: S(G) \otimes_{\gamma} S(H) \rightarrow L^{1}(G) \otimes_{\gamma} L^{1}(H)$, mapping $s \otimes t$ to $s \otimes t$ and extended to the whole space in the usual way, is an algebraic injection.

Let $T \circ I\left(S(G) \otimes_{\gamma} S(H)\right)=S(G \times H)$ and let us norm $S(G \times H)$ by carrying the norm from $S(G) \otimes_{\gamma} S(H)$. Hereafter, we shall not distinguish between $S(G) \otimes_{\gamma} S(H)$ and $S(G \times H)$. Similar identifications will be made between $L^{1}(G) \otimes_{\gamma} L^{1}(H)$ and $L^{1}(G \times H)$. The next result is also from [5].

IV. $S(G \times H)$ has the following properties:

(i) It is a dense ideal in $L^{1}(G \times H)$ and $\|\phi * \psi\|_{s} \leqslant\|\phi\|_{1}\|\psi\|_{s}$ for $\phi \in$ $L^{1}(G \times H)$ and $\psi \in S(G \times H)$. \|\|$_{s}$ denotes the norm on $S(G \times H)$.

(ii) $\|\psi\|_{1} \leqslant K\|\psi\|_{s}$ for every $\psi \in S(G \times H)$, where $K$ is a suitable constant.

(iii) All integrable functions on $G \times H$ whose Fourier transforms have compact supports are contained in $S(G \times H)$ and they form a dense subset of $S(G \times H)$.

In view of IV above, it follows from Cigler [2] that $S(G \times H)$ is a Segal algebra on $G \times H$. The Segal algebra $S(G \times H)$ is called the tensor product of $S(G)$ and $S(H)$. As mentioned earlier, we shall freely use the identifications between $S(G) \otimes_{\gamma} S(H)$ and $S(G \times H)$ and between $L^{1}(G) \otimes_{\gamma} L^{1}(H)$ and $L^{1}(G \times H)$.

3. Main results. In this section, we shall state and prove some results leading to the final result about the existence of a noncompact locally 
compact abelian group $G$ and a Segal algebra $S(G)$ on $G$ such that $M(S(G))$ properly contains $M(G)$.

LEMMA 1. Let $G$ and $H$ be locally compact abelian groups and let $S(G)$ and $S(H)$ be Segal algebras on $G$ and $H$ respectively. Suppose $T_{1} \in M(S(G))$, $T_{2} \in M(S(H))$ and $T=T_{1} \otimes T_{2}$. Then $T \in M\left(S(G) \otimes_{\gamma} S(H)\right)$.

Proof. It is enough to show that $T(f * g)=f * T g$ for $f \in L^{1}(G) \otimes_{\gamma}$ $L^{1}(H)$ and $g \in S(G) \otimes_{\gamma} S(H)$. Suppose $f$ and $g$ are of the form

$$
f=\sum_{1}^{m} p_{j} \otimes q_{j}, \quad g=\sum_{1}^{n} s_{i} \otimes t_{i} .
$$

Then

$$
f * g=\sum_{i, j}\left(p_{j} * s_{i}\right) \otimes\left(q_{j} * t_{i}\right)
$$

Therefore,

$$
\begin{aligned}
T(f * g) & =\sum_{i, j} T_{1}\left(p_{j} * s_{i}\right) \otimes T_{2}\left(q_{j} * t_{i}\right) \\
& =\sum_{i, j}\left(p_{j} * T_{1} s_{i}\right) \otimes\left(q_{j} * T_{2} t_{i}\right) \\
& =\left(\sum_{j} p_{j} \otimes q_{j}\right) *\left(\sum_{i} T_{1} s_{i} \otimes T_{2} t_{i}\right) \\
& =\left(\sum_{j} p_{j} \otimes q_{j}\right) * T\left(\sum_{i} s_{i} \otimes t_{i}\right) \\
& =f * T g .
\end{aligned}
$$

Suppose, now, $f$ and $g$ are arbitrary elements of $L^{1}(G) \otimes_{\gamma} L^{1}(H)$ and $S(G) \otimes_{\gamma} S(H)$ respectively. Let us choose sequences $\left\{f_{n}\right\}$ in $L^{1}(G) \otimes_{\gamma}$ $L^{1}(H)$ and $\left\{g_{n}\right\}$ in $S(G) \otimes_{\gamma} S(H)$ such that $f_{n}$ 's and $g_{n}$ 's are of the form (1) and such that $\left\|f_{n}-f\right\|_{1} \rightarrow 0$ and $\left\|g_{n}-g\right\|_{s} \rightarrow 0$. Then $\left\|f_{n} * g_{n}-f * g\right\|_{s} \rightarrow 0$ and, therefore,

$$
T(f * g)=\lim _{n} T\left(f_{n} * g_{n}\right)=\lim _{n} f_{n} * T g_{n}=f * T g .
$$

This completes the proof.

LEMMA 2. With the same notations as in Lemma 1 , we have $\left(T_{1} \otimes T_{2}\right)^{\wedge}$ $=\hat{T}_{1} \cdot \hat{T}_{2}$.

Proof. Let $\left(\gamma_{1}, \gamma_{2}\right)$ be an arbitrary element of $\hat{G} \times \hat{H}$. For $s \in S(G)$ and $t \in S(H)$, we have

$\left[\left(T_{1} \otimes T_{2}\right)(s \otimes t)\right]^{\wedge}\left(\gamma_{1}, \gamma_{2}\right)=\left(T_{1} s \otimes T_{2} t\right)^{\wedge}\left(\gamma_{1}, \gamma_{2}\right)$

$$
=\left(T_{1} s\right)^{\wedge}\left(\gamma_{1}\right) \cdot\left(T_{2} t\right)^{\wedge}\left(\gamma_{2}\right)=\hat{T}_{1}\left(\gamma_{1}\right) \hat{s}\left(\gamma_{1}\right) \cdot \hat{T}_{2}\left(\gamma_{2}\right) \hat{t}\left(\gamma_{2}\right) .
$$

Also,

$$
\begin{aligned}
{\left[\left(T_{1} \otimes T_{2}\right)(s \otimes t)\right]^{\wedge}\left(\gamma_{1}, \gamma_{2}\right) } & =\left(T_{1} \otimes T_{2}\right)^{\wedge}\left(\gamma_{1}, \gamma_{2}\right) \cdot(s \otimes t)^{\wedge}\left(\gamma_{1}, \gamma_{2}\right) \\
& =\left(T_{1} \otimes T_{2}\right)^{\wedge}\left(\gamma_{1}, \gamma_{2}\right) \cdot \hat{s}\left(\gamma_{1}\right) \hat{t}\left(\gamma_{2}\right) .
\end{aligned}
$$


Choosing $s$ and $t$ in such a way that $\hat{s}\left(\gamma_{1}\right) \neq 0$ and $\hat{t}\left(\gamma_{2}\right) \neq 0$, we get from (2) and (3) that $\left(T_{1} \otimes T_{2}\right)^{\wedge}\left(\gamma_{1}, \gamma_{2}\right)=\hat{T}_{1}\left(\gamma_{1}\right) \cdot \hat{T}_{2}\left(\gamma_{2}\right)$. Thus the lemma is proved.

For a locally compact abelian group $G$, let $B(\hat{G})=\{\hat{\mu}: \mu \in M(G)\}$.

Lemma 3. If $T_{1}, T_{2}$ are as in Lemma 1 and either $\hat{T}_{1} \notin B(\hat{G})$ or $\hat{T}_{2} \notin B(\hat{H})$ and $T_{1} \neq 0$ and $T_{2} \neq 0$, then $\left(T_{1} \otimes T_{2}\right)^{\wedge} \notin B(\hat{G} \times \hat{H})$.

Proof. Let $\phi=\left(T_{1} \otimes T_{2}\right) \hat{\imath}, \phi_{1}=\hat{T}_{1}$ and $\phi_{2}=\hat{T}_{2}$. Then $\phi, \phi_{1}$ and $\phi_{2}$ are continuous. Suppose $\phi_{1} \notin B(G)$. (The arguments in the case when $\phi_{2} \notin$ $B(\hat{H})$ are exactly similar and, hence, omitted.) Since $\phi_{1} \notin B(\hat{G})$ and $\phi_{1}$ is continuous, by Eberlein's characterization [9, Rudin], for every positive integer $n$, there exists a trigonometric polynomial $\sum c_{i} \gamma_{i}$ on $G$ such that $\left|\sum c_{i} \phi_{1}\left(\gamma_{i}\right)\right| \geqslant n\left\|\sum c_{i} \gamma_{i}\right\|_{\infty}$.

Choose $\delta \in \hat{H}$ such that $\phi_{2}(\delta) \neq 0$. Then

$$
\begin{aligned}
\left|\sum c_{i} \phi\left(\gamma_{i}, \delta\right)\right| & =\left|\phi_{2}(\delta)\right|\left|\sum c_{i} \phi_{1}\left(\gamma_{i}\right)\right| \geqslant n\left|\phi_{2}(\delta)\right|\left\|\sum c_{i} \gamma_{i}\right\|_{\infty} \\
& =n\left|\phi_{2}(\delta)\right|\left\|\sum c_{i}\left(\gamma_{i}, \delta\right)\right\|_{\infty} .
\end{aligned}
$$

Again, by Eberlein's characterization, it follows that $\phi \notin B(\hat{G} \times \hat{H})$. This finishes the proof.

The next corollary is an immediate consequence of Lemmas 1-3.

Corollary 1. Let $G, \dot{H}$ be locally compact abelian groups and $S(G)$ and $S(H)$ Segal algebras on $G$ and $H$ respectively. If either $M(S(G))$ properly contains $M(G)$ or $M(S(H))$ properly contains $M(H)$ then $M(S(G \times H))$ properly contains $M(G \times H)$.

Our main result is now very easy to see.

THEOREM. There exists a noncompact locally compact abelian group $G$ and $a$ Segal algebra $S(G)$ on $G$ such that $M(S(G))$ properly contains $M(G)$.

Proof. Let $H$ be a noncompact locally compact abelian group and $K$ an infinite compact abelian group. Let $G=H \times K$ and $S(G)$ $=L^{1}(H) \otimes_{\gamma} L^{2}(K)$. Since $M\left(L^{2}(K)\right)$ properly contains $M(K)$, Corollary 1 implies that $M(S(G))$ properly contains $M(G)$. Thus the theorem is proved.

The following corollary is of interest for $A^{p}$-algebras. For $1 \leqslant p<\infty$ and a locally compact abelian group $G$, we define $A^{p}(G)=\left\{f \in L^{1}(G): \hat{f} \in\right.$ $\left.L^{p}(\hat{G})\right\}$. For $f \in A^{p}(G)$, we define $\|f\|_{A^{p}}=\|f\|_{1}+\|\hat{f}\|_{p}$. Then $A^{p}(G)$ is a Segal algebra. It is well known [7] that $M\left(A_{p}(G)\right)$ is isometrically isomorphic to $M(G)$ for noncompact $G$ and that $M\left(A_{p}(G)\right)$ properly contains $M(G)$ if $G$ is an infinite compact abelian group. In view of this and Corollary 1, we obtain:

COROllary 2. If $G$ is noncompact and $H$ is an infinite compact abelian group, then $A^{p}(G) \otimes_{\gamma} A^{q}(H)$ is not isomorphic to $A^{r}(G \times H)$ for any $r$, $1 \leqslant r<\infty$ and $1 \leqslant p, q<\infty$.

4. Multipliers of the Wiener algebra. In this section we show that the multipliers of $M_{1}(R)$ to $L^{1}(R)$ are not the same as the multipliers of $M_{1}(R)$. 
Proposition 1. $M\left(M_{1}(R), L^{1}(R)\right)$ properly includes $M\left(M_{1}(R)\right)$.

Proof. Edwards [3] has proved that $M\left(M_{1}(R), L^{1}(R)\right)$ is isomorphic to $P^{1}(R)$ where $P^{1}(R)$ is the set of all pseudomeasures $s$ on $R$ such that $s * C_{c}(R) \subset L^{1}(R)$, where $C_{c}(R)$ denotes the space of all continuous functions on $R$ having compact supports. Let $V$ denote the set of all Radon measures $\mu$ on $R$ such that $\sup _{k \in Z}|\mu|([k, k+1])<\infty$, where $Z$ denotes the group of integers; Keshava Murthy and Unni [6] have proved that if $T \in$ $M\left(M_{1}(R)\right)$, then there exists a unique $\mu \in V$ such that $T f=f * \mu$ for every $f \in M_{1}(R)$. Thus we see that $M\left(M_{1}(R)\right) \subset V$. Let $P_{c}(R)$ denote the set of all pseudomeasures on $R$ having compact supports. Edwards [3] had observed that $P_{c}(R) \subset P^{1}(R)$, while it is obvious that $P_{c}(R) \not \subset V$ because $P_{c}(R) \cap V$ $\subset M(R)$, but $P_{c}(R)$ is not contained in $M(R)$. Thus $P_{c}(R)$ is not contained in $M\left(M_{1}(R)\right)$, while $P_{c}(R) \subset M\left(M_{1}(R), L^{1}(R)\right)$. Since it is easily seen that $M\left(M_{1}(R)\right) \subset M\left(M_{1}(R), L^{1}(R)\right)$, it follows that $M\left(M_{1}(R)\right)$ is properly included in $M\left(M_{1}(R), L^{1}(R)\right)$. This completes the proof.

\section{REFERENCES}

1. J. T. Burnham, Multipliers of A-Segal algebras on LCA groups. I (preprint).

2. J. Cigler, Normed ideals in $L^{1}(G)$, Nederl. Akad. Wetensch. Proc. Ser. A $72=$ Indag. Math. 31 (1969), 273-282. MR 40 \#3327.

3. R. E. Edwards, Operators commuting with translations, Pacific J. Math. 16 (1966), 259-265. MR 32 \#6151.

4. G. P. Johnson, Spaces of functions with values in a Banach algebra, Trans. Amer. Math. Soc. 92 (1959), 411-429. MR 21 \#5910.

5. V. V. Kapoor, Tensor products of Segal algebras, Doctoral Dissertation, I.I.T. Kanpur, 1972.

6. G. N. Keshava Murthy and K. R. Unni, Multipliers on a space of Wiener functions, Matscience, Madras, India, 1972 (preprint).

7. R. Larsen, Introduction to the theory of multipliers, Springer-Verlag, Berlin and New York, 1971.

8. H. Reiter, $L^{1}$-algebras and Segal algebras, Lecture Notes in Math., vol. 231, Springer-Verlag, Berlin and New York, 1971.

9. W. Rudin, Fourier analysis on groups, Interscience Tracts in pure and Appl. Math., no. 12, Interscience, New York, 1962. MR 27 \#2808.

Department of Mathematics, Indian Institute of Technology, Kanpur 208016, U.P, INDIA 